\title{
Das Beste für die Patienten
}

Vor einem halben Jahr hat Prof. Dr. med. Claudia Witt die Nachfolge von Prof. Dr. med. Reinhard Saller am Universitätsspital in Zürich angetreten. Sie ist überzeugt davon, dass eine sinnvolle Kombination von Schul- und Komplementärmedizin für chronisch kranke Patienten Nutzen bringt.

Früher stand Naturheilkunde am Klingelschild des Instituts. Seitdem Sie die Leitung übernommen haben, heisst es «Institut für komplementäre und integrative Medizin». Wieso haben Sie sich zu dem Namenswechsel entschlossen?

Prof. Dr. med. Claudia Witt: Weil das, was wir am Institut anbieten, über das hinausgeht, was die klassische Naturheilkunde mit ihren fünf Säulen beinhaltet. Wir berücksichtigen neben der Phytotherapie und anderen Elementen der Naturheilkunde auch Aspekte der Traditionellen Chinesischen Medizin, der Mind-Body-Medizin und der Osteopathie. Die Akupunkturforschung gehört z.B. zu meinen Schwerpunkten. Der Begriff der komplementären und integrativen Medizin charakterisiert unsere Tätigkeit treffender, zumal ja unsere Ärzte auch schulmedizinisch hervorragend ausgebildet sind und wir eng mit anderen Kliniken zusammenarbeiten. Aber eigentlich ist die ganze Diskussion über Terminologie aus meiner Sicht zweitrangig; es geht einfach darum, gute Patientenversorgung, Forschung und Lehre zu machen.

\section{Welche Tendenzen beobachten \\ Sie im Moment in der komplemen- tären und integrativen Medizin?}

Das hängt vom Land ab, über das man redet. Die Entwicklungen in der Komplementärmedizin im deutschsprachigen Raum und beispielsweise in den USA sind durchaus verschieden. In der Schweiz und in Deutschland gibt es eine lange Tradition im Bereich der Phytotherapie, der Homöopathie und der Anthroposophischen Medizin. Die Chinesische Medizin hat in den letzten Jahrzehnten zudem sehr an Bedeutung gewonnen. Wenn man sich z.B. die Verhältnisse in den USA anschaut, wird schnell klar, dass dort andere Dinge eine Rolle spielen als auf der hiesigen Seite des Atlantiks. Dort sind beispielsweise Massagen sehr häufig genutzte Verfahren in der Komplementärmedizin. Auch Entspannungstechniken, aus dem Bereich der Mind-Body-Medizin, werden von Patientinnen und Patienten dort sehr stark nachgefragt. Und nicht $\mathrm{zu}$ vergessen der verwirrende Markt der Nahrungsergänzungsmittel, mit sehr heterogener Qualität. Wenn man über Unterschiede redet, muss man auch darüber reden, wer die Anbieter sind. In der Schweiz wie in Deutschland gibt es auch eine Ärzteschaft, die komplementärmedizinische Verfahren durchführt. In den USA sind es sehr selten Ärzte, sondern zumeist Therapeuten. Diese sind jedoch oft akademisch ausgebildet.

Findet in der Komplementärmedizin ein internationaler Austausch statt oder forscht man eher isoliert?

Vernetzung ist in so einem kleinen Forschungsbereich extrem wichtig, und Kooperation liegt mir sehr am Herzen. International bin ich sehr gut

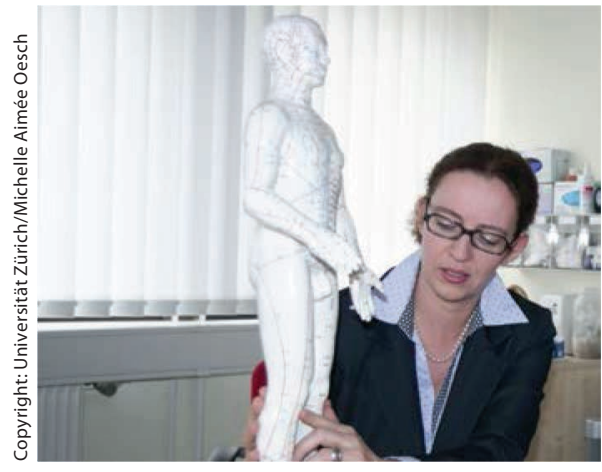

vernetzt und war 2 Jahre lang Präsidentin der International Society for Complementary Medicine Research, deren Ziel die Stärkung der internationalen Zusammenarbeit ist. Die Zusammenarbeit über die Grenzen hinweg und der Umgang mit anderen Kulturen ist das, was die Wissenschaft noch spannender und interessanter macht. Das von der EU geförderte CAMbrella-Projekt, an dem sowohl Professor Saller als auch ich mitgewirkt haben, hat beispielweise die $\mathrm{Zu}$ sammenarbeit in Europa unter den Forschern gefördert.

\section{Wo gibt es derzeit die grösste Nachfrage vonseiten der Patienten?}

Ganz klar im onkologischen Bereich. Das beobachte ich in unserer Poliklinik hier in Zürich wie auch international. Auch in führenden onkologischen Zentren in den USA, wie dem Memorial Sloan-Kettering Cancer Center und dem MD Anderson Cancer Center, hat sich eine Integrative Onkologie etabliert, die

\section{KARGER}

Fax +497614520714 
den Patienten eine zusätzliche komplementärmedizinische Therapie ermöglicht. Dass dieses Thema auch für die Schweiz wichtig ist, zeigt das gut besuchte «Symposium Integrative Onkologie», das jedes Jahr in St. Gallen stattfindet.

Ein zweiter Bereich, der sehr stark im Kommen ist, ist jener der Behandlung chronischer Schmerzen. Hier spielen insbesondere Verfahren der Mind-Body-Medizin und auch die Akupunktur eine wichtige Rolle.

Sowohl in der Onkologie als auch in der Therapie chronischer Schmerzen geht es immer um multimodale Therapien; deshalb ist es ja so wichtig, die Komplementärmedizin sinnvoll mit der konventionellen Therapie zu kombinieren.

\section{Wie wirken sich Fortschritte in zum Beispiel bildgebenden Verfahren in der Neurowissen- schaft auf die Komplementär- medizin aus?}

Solche Dinge haben auch einen Einfluss auf unsere Forschung und sorgen für neue Erkenntnisse. Zum Beispiel nutzen wir auch in der Akupunkturforschung fMRI(funktionelle Magnetresonanztomographie)-Scans. Mithilfe von fMRI sind mittlerweile schon so viele Studien gemacht worden, dass wir diese in einer Metaanalyse zusammenfassen konnten.

\section{Welche anderen technologischen Treiber neben dem Neuroimaging sehen Sie in der Komplementär- medizin?}

Es gibt eine ganze Reihe von Verfahren. Wichtiger als einzelne herauszuheben ist, die Fragestellung nicht aus dem Auge zu verlieren. «Welches Verfahren bringt uns wofür etwas?» ist die wichtige Frage, um zu verhindern, Dinge mitzumachen, nur weil sie gerade hip sind. Man braucht eine klare Forschungsfrage und wählt dann die besten Verfahren aus, um sie zu beantworten.

Ich finde es aber sehr spannend, wie etwa Genomics und Biomarker bei der Untersuchung der Chinesischen Medizin eingesetzt werden. Sie könnten Aufschluss darüber geben, welche biologischen Korrelate hinter den chinesischen Konstitutionstypen und den Syndromdiagnosen stecken.

\section{Bei der Akupunktur interessiert \\ Sie eher der Nachweis der \\ Wirksamkeit oder auch die Frage, wie man sie verbessern kann?}

Die Fragemöglichkeiten sind bei der Akupunktur vielseitig. Eine ungeklärte Frage ist immer noch die, welche Patienten am meisten von der Akupunktur profitieren. In der Medizin ist es ein grosses Thema, heraus-

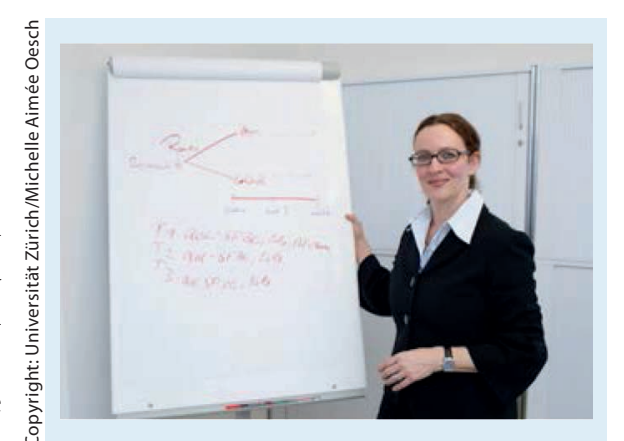

Prof. Dr. med. Claudia Witt, MBA

- 1988-1996: Studium der Humanmedizin an der Freien Universität Berlin und der Ruhr-Universität Bochum, Deutschland.

- 1999: Dissertation an der HumboldtUniversität zu Berlin, Deutschland

- 2006: Habilitation an der CharitéUniversitätsmedizin Berlin, Deutschland, und Lehrbefugnis für die Fächer Sozialmedizin und Epidemiologie

- 2006: Master of Business Administration (MBA) an der Berlin School of Economics and Law, Deutschland

- 2008-2013: Professorin für Komplementärmedizin an der Charité-Universitätsmedizin Berlin, Deutschland

- 2011-2013: Visiting Professor an der University of Maryland School of Medicine, Baltimore, MD, USA

- Seit 2014: Ordentliche Professorin an der Universität Zürich, Schweiz zufinden, weshalb eine Therapie bei dem einen wirkt und dem anderen nicht. Da sind wir sehr nahe an der Personalized Medicine und der Frage, welche Therapie für welche Patienten die beste ist.

Ein weiterer unklarer Aspekt der Akupunktur ist jener der Dosis. Wie viele Akupunkturen braucht es, damit die Behandlung bei chronischen Schmerzen eine langfristige Wirkung zeigt? Viele scheinbar ganz einfache Sachverhalte in der Akupunktur verstehen wir noch nicht: Was macht den spezifischen Effekt der Akupunktur aus? Ist es der Ort, an dem man die Nadel setzt? Ist es die Art, wie man die Nadel stimuliert?

Was die Versorgung betrifft, wird es spannend sein, zu erfahren, welche Therapien man mit der Akupunktur kombinieren muss, um für einen chronisch kranken Patienten das optimale Therapieergebnis zu erreichen.

\section{Was weiss man über den Wirkungs- mechanismus der Akupunktur?}

Wenn man das naturwissenschaftlich betrachtet, kommt es darauf an, über welche Diagnose man spricht. Schmerzmechanismen unterscheiden sich pathophysiologisch von den Mechanismen z.B. einer allergischen Rhinitis. Bei beiden Diagnosen haben wir aber aus klinischen Studien deutliche Hinweise, dass Akupunktur eine spezifische Wirkung hat. Über die möglichen Wirkmechanismen, z.B. lokale und zentrale schmerzhemmende Mechanismen, wissen wir bei chronischen Schmerzen deutlich mehr als bei der allergischen Rhinitis.

\section{Steigert sich durch Forschung die Akzeptanz der Komplementär- medizin bei Vertretern der klassischen Medizin?}

Ich komme aus einem universitären Umfeld, und meine Erfahrung ist: Wenn man gute Forschung macht, 
spricht man unter Wissenschaftlern dieselbe Sprache. Die Barriere ist aus meiner Erfahrung eher zwischen klinischen Forschern und Grundlagenforschern, wo es zum Teil noch am gegenseitigen Verständnis mangelt. Aber man kann schon klar sagen, dass durch Kooperation mit anderen Fachbereichen in gemeinsamen Forschungsprojekten oft erste Schritte einer vertieften Zusammenarbeit entstehen. Deshalb ist es sinnvoll, sich Themen auszuwählen, wo man Interessenüberschneidungen findet. Man darf aber auch nicht vergessen, dass die Stellung von Forschungsthemen und die Qualifikation der Wissenschaftler auch stark von den Rahmenbedingungen der Wissenschaft abhängt. In den USA gibt es sehr gute Rahmenbedingungen in der Komplementärmedizin. Das aber nur deshalb, weil die National Institutes of Health die Professionalisierung dieses Bereichs besonders gefördert haben. Da haben wir in der Schweiz und in Deutschland noch einiges an Strukturförderbedarf.

\section{Den Gegensatz zwischen Komplementärmedizin und klassischer Medizin gibt es in der Forschung dann nicht mehr?}

Nicht wirklich. Eine einfache Antwort auf diese Frage gibt es aber nicht, denn es geht um Nuancen. Nehmen wir mal das Beispiel der Chinesischen Medizin, die ja sehr komplex ist. Ein Patient bekommt ja nicht nur Akupunktur - diese ergänzt normalerweise eine Verschreibung von verschiedenen Kräutern. Im Prinzip wird die Behandlung für jeden Patienten individuell kombiniert.

Von der Forschungsmethodik her ist das deshalb kompliziert: Ich kann nicht wissenschaftlich belegen, was gewirkt hat. Die 15 Akupunkturen, die zur Behandlung gehörten? Die drei Verschreibungen von Kräutern? Welches der Kräuter? Das macht deutlich, dass wir über komplexe

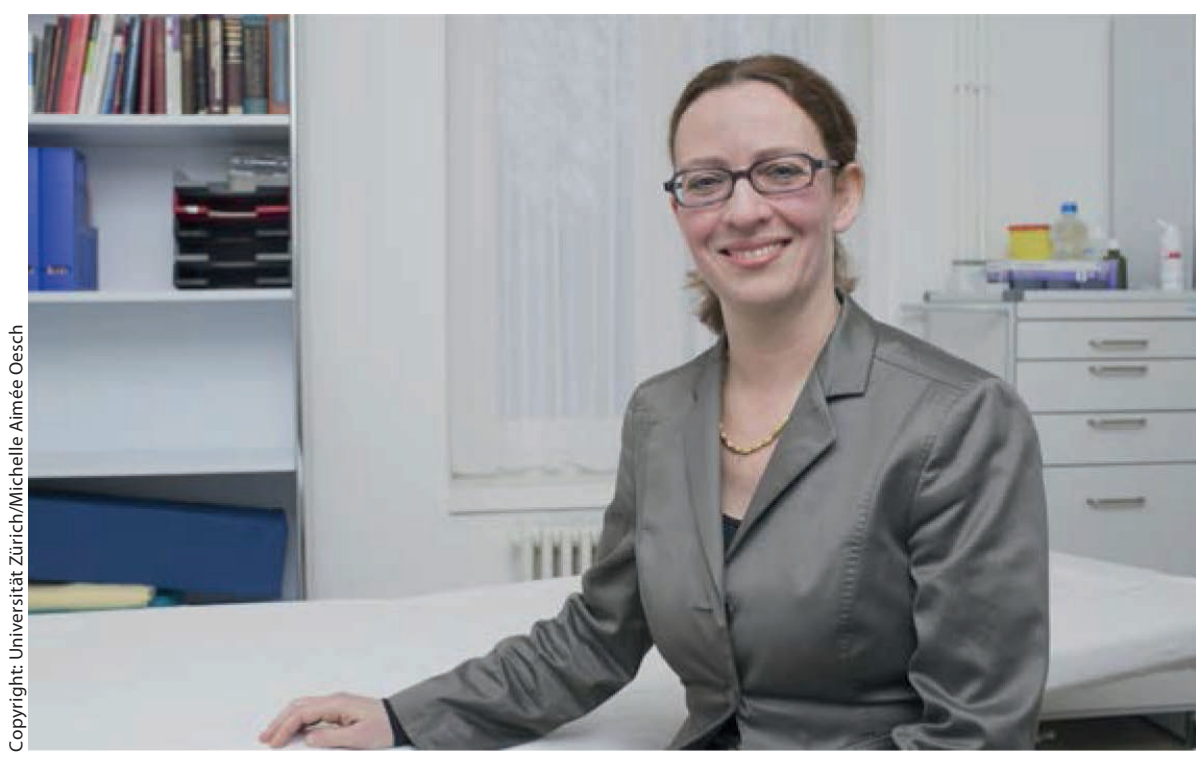

Interventionen sprechen, die nicht so einfach zu evaluieren sind.

Das gleiche Problem haben wir bei der Evaluation einer Reha-Behandlung von beispielweise chronischen Schmerzen. Wenn wir dort Physiotherapie mit Schmerzmedikation kombinieren, ist es auch komplex. Was ich damit meine, ist, dass komplexe Interventionen immer schwieriger zu erforschen sind als Einzelinterventionen und dass dies nicht nur in der Komplementärmedizin der Fall ist.

Was wirklich ein Unterschied ist, ist die Individualisierung in der Therapie. Das hat in der Komplementärmedizin Tradition und wird in der konventionellen Medizin durch die Personalized Medicine gerade erst wieder entdeckt.

\section{Was ist Ihre Motivation, Komple- mentärmedizin zu betreiben?}

Mich hat dieses Feld schon immer gereizt. Das hat damit zu tun, dass der Mensch nicht fragmentiert wahrgenommen wird. Es wird versucht, ihn in der Gesamtheit seiner Symptome, aber auch mit der Gesamtheit seiner Ressourcen $\mathrm{zu}$ sehen und $\mathrm{zu}$ verstehen. Deshalb sind für mich auch die Aspekte der Lebensführung wichtig. In der Naturheilkunde ist es die
Ordnungstherapie und im angloamerikanischen Bereich die MindBody-Medizin. Auch die besondere Art der Arzt-Patienten-Interaktion ist sehr inspirierend. Empathie ist nur ein Element davon.

Ich bin sehr schnell durch die Forschungsfragen motiviert worden. Nachdem ich erst einmal mit der Forschung angefangen hatte, konnte und wollte ich einfach nicht mehr aufhören. Gerade die Kombination von Forschung mit der Möglichkeit, für Patienten Sinnvolles zu tun, gefällt mir.

\section{Welche Rolle spielt für Sie in Ihrem ärztlichen Verständnis das Heilen im Sinne von «wieder ganz gesund machen»?}

In unserer Poliklinik haben wir oft mit schwer kranken Menschen zu tun, bei deren Behandlung man mit dem Anspruch des «Wieder-gesund-Machens» rasch an seine Grenzen stösst. Man kann die Patienten begleiten und ihre Ressourcen stärken, ihnen dabei helfen, ihren Zustand zu verbessern, oder anstreben, dass sie einfach weniger Nebenwirkungen haben. Das ist gerade in der Onkologie besonders wichtig, wenn Menschen durch eine sehr schwere Zeit in der Erkrankung gehen. 
Es ist das Begleiten der Patienten, das Verbessern der Situation und das "Erträglicher-Machen», das die Befriedigung an der klinischen Arbeit gibt?

Ja, aber es kommt noch eine wichtige ärztliche Aufgabe hinzu. Ich glaube, es ist z.B. für einen Krebspatienten eine grosse Hilfe, wenn der Arzt ihm hilft, das Angebot in diesem Bereich zu sortieren. Es ist ja eine verwirrende Vielfalt, die dort auf den Patienten einstürzt und mit der er konfrontiert wird: Da kommt der Nachbar mit einem Tipp. Da warten Verwandte mit einem anderen auf, und die Patienten finden im Internet die unterschiedlichsten Informationen. Sie befinden sich in einer gesundheitlichen Extremsituation und sind oft komplett überfordert, die Information einzuordnen. Da ist die Bedeutung des Experten, der sich schulmedizinisch und komplementärmedizinisch gut auskennt und der zusammen mit dem Patienten eine Vorauswahl an Verfahren trifft, für die es gewisse wissenschaftliche Belege gibt, gar nicht hoch genug einzuschätzen. Ich glaube, dass diese Hilfestellung für den Patienten eine grosse Erleichterung ist.

Das entspricht auch dem modernen Selbstverständnis und der Rolle des Arztes in einem Shared-DecisionMaking-Prozess, der wichtig für eine gute Arzt-Patienten-Interaktion ist: Man schaut sich die Fakten zusammen an, und dann wird gemeinschaftlich die beste Entscheidung für den Einzelfall getroffen.
Welche Schwerpunkte werden Sie in den nächsten Jahren setzen?

Die bisherigen Schwerpunkte in meinen Forschungsbereichen werde ich beibehalten. Ich werde auf jeden Fall die Akupunkturforschung fortsetzen. Dazu gehören auch meine Kooperationen in den USA und in Deutschland, wie etwa die mit dem Max-Planck-Institut in Leipzig. Auch meine Forschungen $\mathrm{zu}$ nichtmedikamentösen Verfahren im Schmerzbereich werde ich fortführen. Onkologische Indikationen werden eine stärkere Rolle als vorher spielen; dabei interessieren mich insbesondere die Effektivität von Entspannungsverfahren, Yoga, Qi Gong und andere Aspekte aus dem Bereich der MindBody-Medizin.

Dadurch, dass ich als Methodikerin aber eine gute Grundlage besitze, mit der sich viele Fragestellungen beantworten lassen, bin ich thematisch nicht so eng festgelegt. Was die Themen betrifft, sind wir deshalb auch offen für die Interessen unserer Kooperationspartner.

\section{Wo sehen Sie für die Patienten in den nächsten Jahren die grössten Chancen für Verbesserung in der Komplementärmedizin?}

Ich bin überzeugt, dass es für die Patienten die grösste Verbesserung gibt, wenn es zu einem engen $\mathrm{Zu}-$ sammenwirken von Komplementärmedizin und konventioneller Medizin kommt. Ganz wichtig ist auch, dass wir verstehen, welche Informationen $\mathrm{zu}$ dem Thema nötig sind, und dass diese dann auch verlässlich sind. Das Forschungsprojekt der Deutschen Krebshilfe zur Komplementärmedizin in der Onkologie (www.kompetenznetz-kokon.de), an dem ich massgeblich beteiligt bin, wird dazu sicherlich neue Standards setzen.

\section{Man sollte also nicht die Hoffnung auf neue weltbewegende Verfahren setzen, sondern auf die geschickte Kombination von Wissen aus der Schul- und Komplementärmedizin?}

Ja, denn am Horizont sehe ich keine weltbewegenden wissenschaftlichen Neuentwicklungen, die die Medizin vollkommen verändern werden. Ich glaube auch nicht, dass wir noch in irgendwelchen Wäldern die Wunderpflanze finden werden, die die Welt vor Krebs retten wird. Der grösste Nutzen, den wir als Ärzte für unseren Patienten erbringen können, ist, ihnen die beste auf sie zugeschnittene Therapie zu ermöglichen. Dafür müssen wir einfach mehr darüber wissen, was bei wem am besten wirkt, und nicht nur, was im Durchschnitt am besten wirkt. Und wir sollten nicht vergessen, dass eine gute Arzt-Patienten-Interaktion ein wichtiger Schlüssel für eine erfolgreiche Therapie ist.

Interview: Oliver Klaffke 